\title{
A Novel Cost Effective Demosaicing Approach
}

\author{
R. Lukac, K.N. Plataniotis, D. Hatzinakos, and M. Aleksic
}

\begin{abstract}
A unique color interpolation approach for digital still cameras is introduced and described in terms of color vectors. A new difference plane model is introduced and used in conjunction with the proposed here correction process. This avoids edge blurring while improving on the color appearance of previous methods. Experimental results indicate that the proposed method exhibits superior performance over state of-the-art color interpolation methods ${ }^{l}$.
\end{abstract}

Index Terms - Camera image processing, Bayer pattern, color filter array interpolation, color image restoration.

\section{INTRODUCTION}

$\mathrm{C}$ OLOR image processing has aroused much interest and acclaim over the past few years. The advances in hardware and software platforms have allowed capturing and reproducing of real scenes in color as never before. Digital cameras for still images are among the most popular acquisition devices, whose commercial proliferation has a significant impact on the research in this area.

Digital color cameras acquire color information by transmitting the image through Red (R), Green $(\mathrm{G})$ and Blue (B) color filters having different spectral transmittances and then sampling the resulted images using three electronic sensors, usually charge coupled devices (CCD) and complementary metal oxide semiconductor (CMOS) sensors (Fig. 1a). To reduce cost and complexity, digital camera manufacturers use a single CCD/CMOS sensor with a color filter array (CFA) to capture all the three primary colors (R,G,B) at the same time (Fig. 1b). The Bayer pattern (Fig. 2) [1], a widely used CFA, provides the array or mosaic of the RGB colors so that only one color element is available in each pixel, whereas two missing colors must be estimated from the adjacent pixels. This process is called CFA interpolation, or demosaicing [2].

In this paper, we introduce a new CFA interpolation method along with a novel vector notation of interpolation steps. The proposed edge-sensing method employs a new correlationcorrection process based on a simple difference plane model. The introduced vector notation finds a support in fundamentals of color image filtering [3], and allows to describe the CFA interpolation as a compact framework.

\footnotetext{
${ }^{1}$ Manuscript received October 9, 2003.

The authors are with The Edward S. Rogers Sr. Department of ECE, University of Toronto, Canada.

Corresponding Author: Dr. Rastislav Lukac, Bell Canada Multimedia Laboratory, Room BA 4157, The Edward S. Rogers Sr. Department of ECE, University of Toronto, 10 King's College Road, Toronto, Ontario, M5S 3G4, Canada (e-mail: lukacr@ieee.org)
}

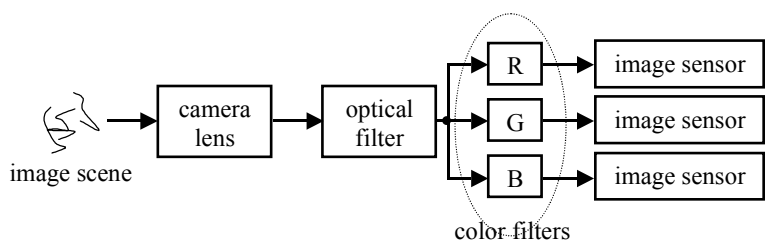

(b)

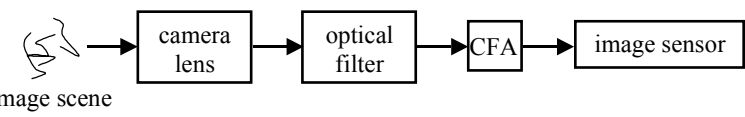

Fig. 1. Digital camera systems: (a) a three-sensor device, (b) a singlesensor device.

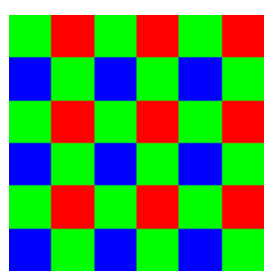

Fig. 2. Bayer CFA pattern.

\section{Problem Formulation}

Let us consider, a $K_{1} \times K_{2}$ gray-scale image $z(i): Z^{2} \rightarrow Z$ representing a two-dimensional matrix of integer samples. In the Bayer CFA pattern, half of the pixels $z_{i}$, for $i=1,2, \ldots, K_{1} K_{2}$, correspond to the $\mathrm{G}$ channel, whereas the R,B channels are assigned the other half of the pixels. Assuming that $p=1,2, \ldots, K_{1}$ and $p=1,2, \ldots, K_{2}$ denote the spatial position of the pixels in vertical (image rows) and horizontal (image columns) directions, gray-scale pixels $z_{i}$ can be transformed into the RGB vectors $\mathbf{x}_{i}=\left(x_{i 1}, x_{i 2}, x_{i 3}\right)$, for $i=(p-1) K_{2}+q$, as follows:

$\mathbf{x}_{i}= \begin{cases}\left(z_{i}, 0,0\right) & \text { for } p \text { odd and } q \text { even, } \\ \left(0,0, z_{i}\right) & \text { for } p \text { even and } q \text { odd, } \\ \left(0, z_{i}, 0\right) & \text { otherwise }\end{cases}$

This transformation forms a $K_{1} \times K_{2}$ RGB image $\mathbf{x}(i): Z^{2} \rightarrow Z^{3}$ representing a two-dimensional matrix of threecomponent samples. Note that the color vectors $\mathbf{x}_{i}$ relate to one true component varying in $k$ from position to position, whereas other two components of $\mathbf{x}_{i}$ are set to zero.

To estimate the missing color components of $\mathbf{x}(i)$, a sliding supporting window $W=\left\{\mathbf{x}_{i} ; i=0,1, \ldots, N-1\right\}$ of finite size $N$ is considered with the sample under consideration, sample $\mathbf{x}_{0}$, placed in the center of the window (Fig. 3). The procedure replaces $\mathbf{x}_{0}$ by some function of the local neighborhood are 


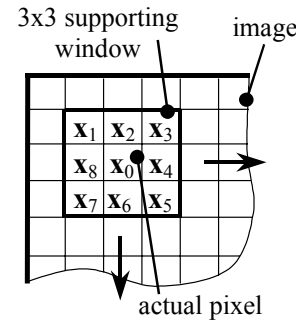

Fig. 3. Sliding supporting window.

$\mathbf{x}_{1}, \mathbf{x}_{2}, \ldots, \mathbf{x}_{N-1}$ at a time. The rationale of this approach is to minimize the local distortion and ensure the stationarity of the processes generating the image [3].

Interpolating the missing color components of the image $\mathbf{x}(i)$ constitutes the interpolated RGB image $\mathbf{y}(i): Z^{2} \rightarrow Z^{3}$. The objective of the interpolation process is to estimate the missing color components of $\mathbf{x}(i)$ and reconstitute the interpolated RGB image $\mathbf{y}(i)$ to be as close as possible to the desired RGB $\mathbf{o}(i): Z^{2} \rightarrow Z^{3}$. As in most image processing problems, a "loss function," which depends on the unavailable full RGB sample and the interpolation vector, is use to penalize errors during the procedure. It is natural to assume that if one penalizes interpolation errors through loss function, then the optimal solution is the function of the inputs that minimizes the expected average loss:

minimize $E\left\{\|\mathbf{o}-\mathbf{y}\|^{2}\right\}$

where $E\{\cdot\}$ indicates statistical expectation guaranteeing the minimum average loss or risk [2].

\section{Proposed METhod}

It is known that the human visual system is sensitive to changes in color and edge information, which provides indication of the shape of objects in the image [3]. False colored and blurred edges introduced as result of inaccurate interpolation significantly degrade the quality of the perceived image. Therefore, efficient interpolation algorithms incorporate edge information into the interpolation process.

A number of edge-detection mechanisms, which differentiate in preferred edge directions, have been introduced in the past years. The proposed method takes advantage of an 8-direction system of [4], which determines edge-sensing weight coefficients as follows:

$$
\begin{aligned}
& w_{1}=\frac{1}{1+\left(\left|z_{0}-z_{9}\right|+\left|z_{1}-z_{5}\right|\right) /(2 \sqrt{2})} \\
& w_{2}=\frac{1}{1+\left(\left|z_{0}-z_{11}\right|+\left|z_{2}-z_{6}\right|\right) / 2}
\end{aligned}
$$

where $w_{1}$ and $w_{2}$ denote weights in north-west and north directions (Fig 4) and $z_{0}, z_{1}, \ldots, z_{24}$ are original gray-scale values of $z(i)$. Weights $w_{2}, w_{3}, \ldots, w_{8}$ are calculated analogously to (3) and (4). Note that $N=9$ relates to a $3 \times 3$ sliding window, $z_{0}$ is the acquired pixel before the

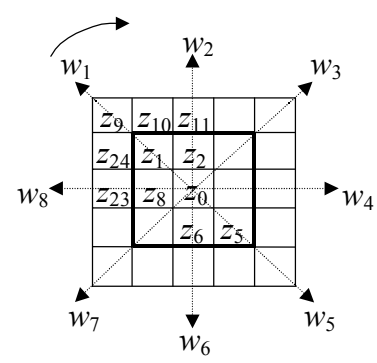

Fig. 4. Edge-sensing scheme used in the proposed design.

transformation (1) in the same spatial position as the RGB vector $\mathbf{x}_{i}$ and operator $\cong$ denotes a one to one relationship.

Using the edge-sensing scheme shown in Fig. 4, the G channel is adaptively interpolated as follows:

$\bar{y}_{02}= \begin{cases}x_{02} & \text { if } z_{0} \cong x_{0 k}, \\ \sum_{i=1}^{N-1} w_{i}^{\prime} x_{i 2}^{\prime} & \text { otherwise }\end{cases}$

where $w_{i}^{\prime}$, for $i=1,2, \ldots, 8$, are the normalized weighting coefficients, given by

$w_{i}^{\prime}=w_{i} / \sum_{j=1}^{N-1} w_{j}$

Each $w_{i}^{\prime}$ corresponds to the pre-determined G component $x_{i 2}^{\prime}$ of [4]. For illustration purposes, $x_{(1) 2}^{\prime}$ and $x_{(2) 2}^{\prime}$ corresponding to the weighting coefficients of (3) and (4) are given by

$x_{(1) 2}^{\prime}=\frac{x_{(2) 2}+x_{(8) 2}+\left(z_{1}-z_{5}\right) /(2 \sqrt{2})+\left(z_{11}-z_{0}+z_{23}-z_{0}\right) / 4}{2}$
$x_{(2) 2}^{\prime}=x_{(2) 2}+\left(z_{11}-z_{0}+z_{2}-z_{6}\right) / 4$

The edge sensitivity is controlled by the weighting coefficients $w_{i}$, for $i=1,2, \ldots, 8$. When no edge is positioned across the directions considered by $x_{i 2}^{\prime}$, the absolute difference used in the corresponding weight $w_{i}$ is small and thus $x_{i 2}^{\prime}$ contributes significantly to the estimated output of (5). If $x_{i 2}^{\prime}$ is defined across an edge, the corresponding $w_{i}$ increases and penalizes $x_{i 2}^{\prime}$ appropriately. Since the solution is unbiased [3] i) each weight is a positive number, $w_{i}^{\prime} \geq 0$, and ii) the summation of all the weights is equal to unity:

$$
\sum_{i=1}^{N-1} w_{i}=1
$$

Interpolating the $\mathrm{G}$ components, we can operate on more information compared to whose of the original Bayer pattern. Since the G components determine for the most part the perceived sharpness of the digital image, adopting the colordifference model of [5], and assuming the stationarity of the processes inside the local image area of $W$, the missing $\mathrm{R}$ (or B) components of $\mathbf{x}(i)$ are given by 


$$
\bar{y}_{0 k}= \begin{cases}x_{0 k} & \text { if } z_{0} \cong x_{0 k}, \\ \bar{y}_{02}+\sum_{i=1}^{(N-1) / 2} w_{i}^{\prime \prime}\left(x_{(2 i) k}-\bar{y}_{(2 i) 2}\right) & \text { if } z_{0} \cong x_{02}, \\ \bar{y}_{02}+\sum_{i=1}^{(N-1) / 2} w_{i}^{\prime \prime \prime}\left(x_{(2 i-1) k}-\bar{y}_{(2 i-1) 2}\right) & \text { if } z_{0} \cong x_{0(k \pm 2)},\end{cases}
$$

where $k=1$ and $k=3$ characterize the $\mathrm{R}$ and $\mathrm{B}$ channels, respectively and $w_{1}^{\prime \prime}, w_{2}^{\prime \prime}, \ldots, w_{4}^{\prime \prime}$ are the normalized weights corresponding to edges in north, east, south and west directions, whereas $w_{1}^{\prime \prime \prime}, w_{2}^{\prime \prime \prime}, \ldots, w_{4}^{\prime \prime \prime}$ are the weights corresponding to the diagonally positioned edges:

$$
\begin{aligned}
& w_{i}^{\prime \prime}=w_{(2 i)} / \sum_{j=1}^{(N-1) / 2} w_{(2 j)} \\
& w_{i}^{\prime \prime \prime}=w_{(2 i-1)} / \sum_{j=1}^{(N-1) / 2} w_{(2 j-1)}
\end{aligned}
$$

Since (10) produces all color components and results in the restored color image, the basic idea of (10) can be used as a correction step. Introducing a correction mechanism into the interpolation process improves contrast and accuracy of the initially interpolated $G$ channel. Since the difference plane model of (6) is simple to implement, it is also perfectly suited to a correction stage. Thus, the correction process related to the $\mathrm{G}$ channel is given by

$$
y_{02}= \begin{cases}\bar{y}_{0 k}+\sum_{i=1}^{(N-1) / 2} w_{i}^{\prime \prime}\left(\bar{y}_{(2 i) 2}-\bar{y}_{(2 i) k}\right) & \text { if } z_{0} \cong x_{0 k}, \\ \bar{y}_{02} & \text { otherwise }\end{cases}
$$

where $w_{1}^{\prime \prime}, w_{2}^{\prime \prime}, \ldots, w_{4}^{\prime \prime}$ are the same coefficients used in (6).

Considering the corrected $G$ values of (13) the R,B update is completed using the following step:

$$
y_{0 k}= \begin{cases}y_{0 k} & \text { if } z_{0} \cong x_{0 k}, \\ y_{02}+\sum_{i=1}^{(N-1) / 2} w_{i}^{\prime \prime}\left(\bar{y}_{(2 i) k}-y_{(2 i) 2}\right) & \text { if } z_{0} \cong x_{02}, \\ y_{02}+\sum_{i=1}^{(N-1) / 2} w_{i}^{\prime \prime \prime}\left(\bar{y}_{(2 i-1) k}-y_{(2 i-1) 2}\right) & \text { if } z_{0} \cong x_{0(k \pm 2)},\end{cases}
$$

Since $x_{0 k}$ of (1), $\bar{y}_{0 k}$ of (5) and (10); and $y_{0 k}$ of (13) and (14), for $z_{0} \cong x_{0 k}$ and $k=1,2,3$, are equivalent, the proposed edge-sensing correlation-correction (ESCC) method preserves all acquired (correct) values of (1) unchanged:

$$
y_{i k}=x_{i k}, \text { if } z_{i} \cong x_{i k}
$$

leading to an efficient and attractive method.
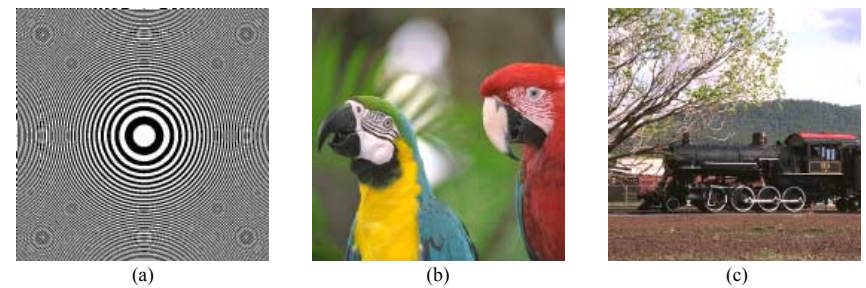

Fig. 5. Test images: (a) circular zone plate (CZP) image, (b) color image Parrots, (c) color image Train
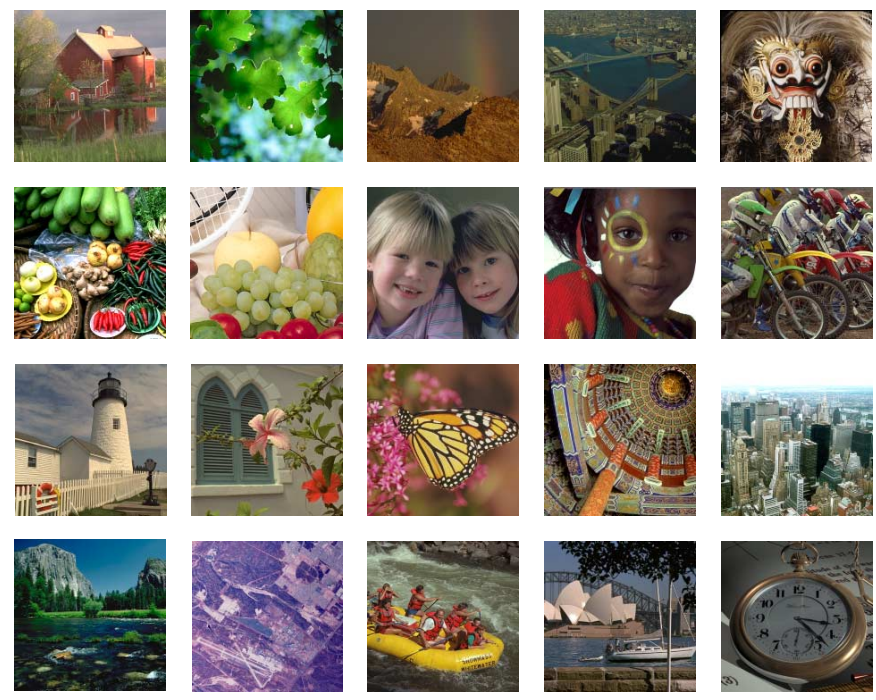

Fig. 6. Color image database.

\section{EXPERIMENTAL RESULTS}

The proposed method is tested using the color test images shown in Fig. 5 and Fig. 6. Note that circular zone plate (CZP) image shown in Fig. 5a is a synthetic black and white image. Using this image researchers examine the performance of the interpolation methods for various edge frequency. Fig. 5b,c and Fig. 6 show the color images that have been captured using highly professional three-sensor cameras or color scanners and they have been extensively used by practitioners and researchers working in camera image processing, especially in demosaicing. This mainly due to the fact that the database: i) contains natural, real life images, ii) represents real-life scenarios and, iii) its images vary in complexity and color appearance. Note that in order to facilitate comparisons, all images have been normalized to the standard $512 \times 512,8$ bit per channel RGB representation.

To measure the efficiency of the CFA interpolation methods objectively, a $K_{1} \times K_{2}$ original color image $\mathbf{o}(i)$ is transformed into a $K_{1} \times K_{2}$ Bayer image $z(i)$ as follows:

$z_{i}= \begin{cases}o_{i 1} & \text { for } p \text { odd and } q \text { even } \\ o_{i 3} & \text { for } p \text { even and } q \text { odd } \\ o_{i 2} & \text { otherwise }\end{cases}$

where $\mathbf{o}_{i}=\left(o_{i 1}, o_{i 2}, o_{i 3}\right)$ denotes the color vector in the original color image $\mathbf{o}(i)$ with spatial coordinates 
TABLE I

COMPARISON OF THE METHODS USING THE CZP IMAGE

\begin{tabular}{|c|c|c|c|c|}
\hline Method & $\mathrm{MSE}_{\mathrm{R}}$ & $\mathrm{MSE}_{\mathrm{G}}$ & $\mathrm{MSE}_{\mathrm{B}}$ & NCD \\
\hline $\mathrm{AP}$ & 110.01 & 51.21 & 111.35 & 0.2842 \\
\hline API & 506.39 & 236.89 & 516.68 & 0.3869 \\
\hline BI & 3804.12 & 1775.17 & 3849.09 & 0.8433 \\
\hline $\mathrm{BD}$ & 408.78 & 180.29 & 413.30 & 0.4237 \\
\hline $\mathrm{C} 2 \mathrm{D} 2$ & 319.33 & 553.62 & 326.10 & 0.3440 \\
\hline EMI & 970.77 & 319.45 & 916.57 & 0.3682 \\
\hline MFI & 984.47 & 135.13 & 997.33 & 0.4332 \\
\hline Proposed & 78.06 & 78.58 & 78.05 & 0.2281 \\
\hline
\end{tabular}

TABLE II

COMPARISON OF THE METHODS USING THE PARROTS IMAGE

\begin{tabular}{crrrc}
\hline \hline Method & MSE $_{\mathrm{R}}$ & MSE $_{\mathrm{G}}$ & MSE $_{\mathrm{B}}$ & $\mathrm{NCD}$ \\
\hline AP & 4.58 & 3.80 & 8.151 & 0.0167 \\
API & 5.40 & 4.28 & 7.895 & 0.0173 \\
BI & 34.85 & 14.93 & 38.251 & 0.0262 \\
BD & 4.87 & 4.05 & 8.415 & 0.0170 \\
C2D2 & 5.44 & 5.24 & 7.099 & 0.0162 \\
EMI & 20.34 & 17.69 & 10.553 & 0.0207 \\
MFI & 7.09 & 2.90 & 10.433 & 0.0172 \\
Proposed & 3.90 & 2.49 & 6.261 & 0.0152 \\
\hline
\end{tabular}

characterized by $i$. Since the original Bayer image is usually unavailable researchers resort to the approach of (16) in order to obtain test Bayer image data used for the CFA interpolation comparative evaluation [6],[7].

The efficiency of the interpolation methods is measured, objectively, via the mean square error (MSE) and the normalized color difference (NCD) criterion [3]. The MSE measure, which allows the evaluation in the RGB domain, is defined as follows:

$$
M S E_{k}=\frac{1}{K_{1} K_{2}} \sum_{i=1}^{K_{1} K_{2}}\left(o_{i k}-y_{i k}\right)^{2}, \text { for } k=1,2,3
$$

where $\mathbf{o}_{i}=\left(o_{i 1}, o_{i 2}, o_{i 3}\right)$ is the original pixel, $\mathbf{y}_{i}=\left(y_{i 1}, y_{i 2}, y_{i 3}\right)$ is the restored pixel, $i$ denotes the pixel position in a $K_{1} \times K_{2}$ color image and $k$ characterizes the color channel.

The NCD criterion quantifies the perceptual similarity between the original and the obtained solution:

$$
N C D=\frac{\sum_{i=1}^{K_{1} K_{2}} \sqrt{\left(L_{\mathbf{o}_{i}}-L_{\mathbf{y}_{i}}\right)^{2}+\left(u_{\mathbf{o}_{i}}-u_{\mathbf{y}_{i}}\right)^{2}+\left(v_{\mathbf{o}_{i}}-v_{\mathbf{y}_{i}}\right)^{2}}}{\sum_{i=1}^{K_{1} K_{2}} \sqrt{\left(L_{\mathbf{o}_{i}}\right)^{2}+\left(u_{\mathbf{o}_{i}}\right)^{2}+\left(v_{\mathbf{o}_{i}}\right)^{2}}}
$$

where $L$ represents lightness values and $u, v$ chrominance values corresponding to $\mathbf{o}_{i}$ and restored $\mathbf{y}_{i}$ samples expressed in CIELUV color space [3].

The enlarged outputs are obtained using the proposed CFA interpolation approach. Corresponding results are compared with of those achieved using such as bilinear interpolation (BI) [8], bilinear difference (BD) approach [6], alternating projections (AP) approach [7], color correlation-directional derivatives
TABLE III

COMPARISON OF THE METHODS USING THE TRAIN IMAGE

\begin{tabular}{crrrr}
\hline \hline Method & MSE $_{\mathrm{R}}$ & MSE $_{\mathrm{G}}$ & MSE $_{\mathrm{B}}$ & \multirow{2}{*}{$\mathrm{NCD}$} \\
\hline AP & 44.43 & 30.80 & 78.39 & 0.0560 \\
API & 109.24 & 96.62 & 145.01 & 0.0776 \\
BI & 517.71 & 254.59 & 607.19 & 0.1450 \\
BD & 74.71 & 65.45 & 113.47 & 0.0744 \\
C2D2 & 93.30 & 113.51 & 128.97 & 0.0702 \\
EMI & 159.42 & 152.53 & 195.69 & 0.0845 \\
MFI & 115.44 & 46.03 & 163.34 & 0.0798 \\
Proposed & 40.44 & 31.24 & 71.69 & 0.0503 \\
\hline
\end{tabular}

TABLE IV

AVERAGED RESULTS RELATED TO THE COLOR IMAGES SHOWN IN FIG. 6.

\begin{tabular}{crrrr}
\hline \hline Method & MSE $_{\mathrm{R}}$ & MSE $_{\mathrm{G}}$ & MSE $_{\mathrm{B}}$ & \multirow{2}{*}{$\mathrm{NCD}$} \\
\hline AP & 42.07 & 21.93 & 39.91 & 0.0532 \\
API & 51.78 & 32.54 & 50.26 & 0.0580 \\
BI & 154.12 & 72.11 & 159.33 & 0.0883 \\
BD & 44.19 & 27.27 & 45.79 & 0.0570 \\
C2D2 & 44.10 & 34.85 & 45.75 & 0.0537 \\
EMI & 91.48 & 47.95 & 69.97 & 0.0649 \\
MFI & 56.30 & 22.56 & 58.27 & 0.0586 \\
Proposed & 36.55 & 18.87 & 35.80 & 0.0480 \\
\hline
\end{tabular}

method (C2D2) [4], median filter interpolation (MFI) [9], high definition color interpolation scheme (EMI) [10] and adaptive color plane interpolation (API) [11], respectively.

Table I summarizes the results obtained using the artificial CZP image shown in Fig. 5a. It can be seen that the AP scheme and especially the proposed method produce the best results among the tested methods. Applying the methods to the real-life images such as Parrots and Train, the proposed method clearly outperforms others CFA interpolation schemes, as is shown in Table II and Table III.

Table IV summarizes the results corresponding to the image database shown in Fig. 6. Using 20 color images, which vary in color and complexity, the robustness of the methods is examined here. The corresponding averaged results clearly indicate that the proposed method is robust and efficient, improves the image restoration process in terms of objective image quality measures and provides restored image which are impressively similar to the original.

Fig. 7 allows the visual (subjective) evaluation of the methods via the zoomed parts of the restored images. It can be seen that many demosaicing approaches (Fig. 7b-f) result in color artifacts and produce blurred edges. This inaccuracy in estimates is significantly reduced interpolating the images with the proposed scheme (Fig. 7g).

Summarizing the results presented above, the following conclusions can be stated: (i) the proposed method restores the color images in a sharp shape, (ii) the difference of the color appearance between the original image and the images restored by the proposed method is in unperceived form to humans, (iii) the proposed method achieves significant improvement in terms of objective (more than 20-30\%) and subjective evaluation of the image quality than the state of-theart CFA interpolation methods. 
(a)

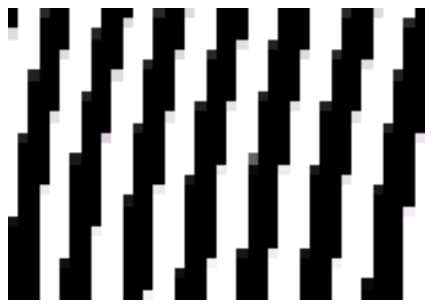

(b)

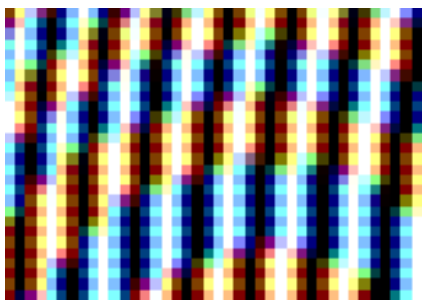

(c)

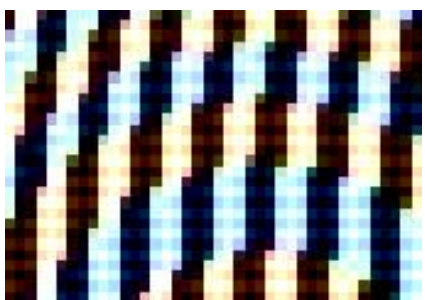

(d)

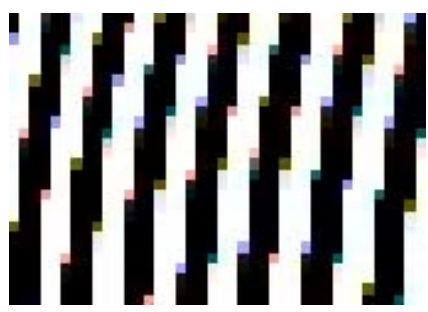

(e)

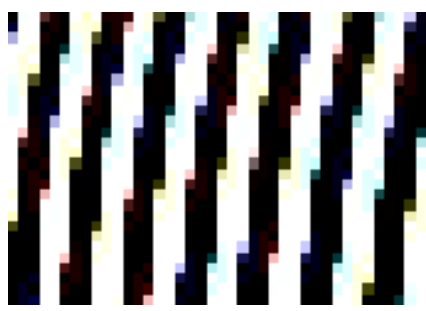

(f)

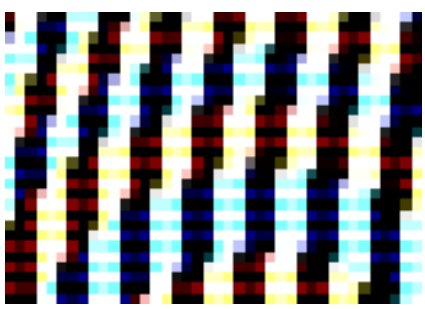

(g)

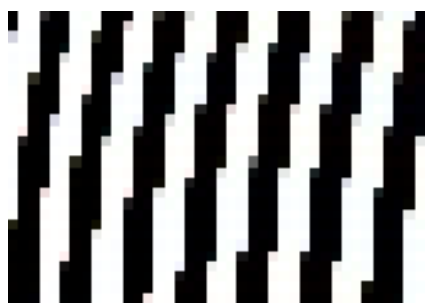

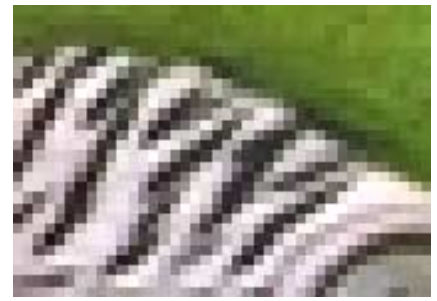
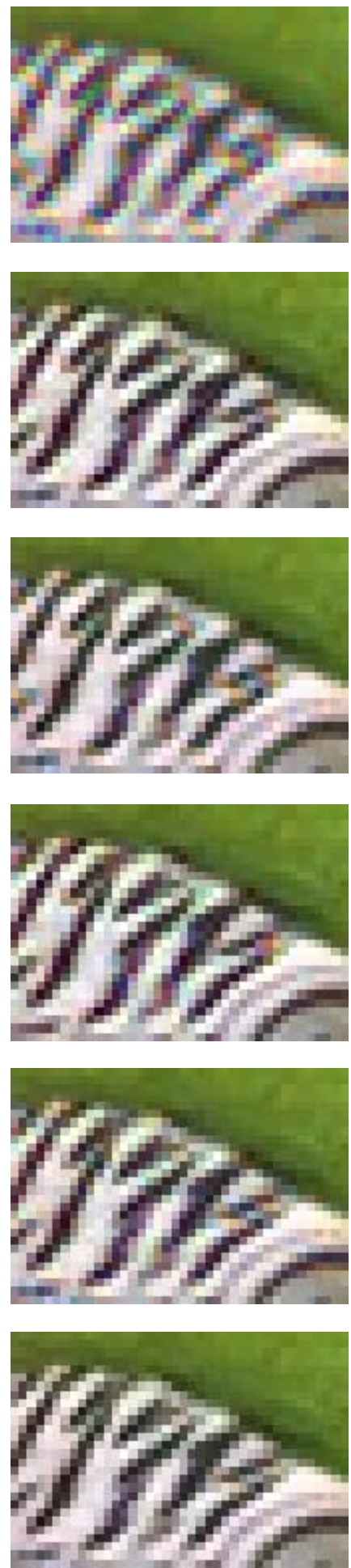
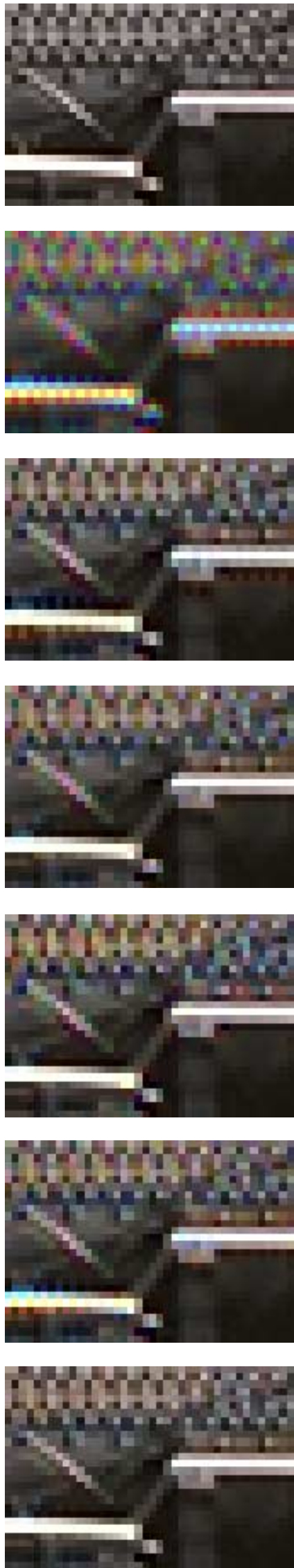

Fig. 7. Zoomed parts of the images corresponding to Fig. 5: (a) original images, (b) BI output, (c) BD output, (d) C2D2 output, (e) API output, (f) MFI output, (g) proposed method output. 


\section{Conclusion}

A novel CFA interpolation approach was presented. The method employed an adaptive edge sensing mechanism, a color difference model and a correction step. Combining their advantages, the proposed method makes the interpolated images sharp, naturally colored and pleasurable for viewing. This also yields excellent results in terms of commonly used objective criteria. Simulation results and comparison reported here indicate that the introduced method is sufficiently robust and significantly outperforms the previously published approaches.

\section{ACKNOWLEDGMENT}

The work of the first author is supported by a NATO/NSERC Science award.

\section{REFERENCES}

[1] B.E. Bayer, "Color imaging array," U.S. Patent 3971 065, 1976.

[2] H.J. Trussell and R.E. Hartwig, "Mathematics for demosaicking," IEEE Trans. Image Processing, vol. 11, no. 4, pp. 485-492, April 2002.

[3] K.N. Plataniotis and A.N. Venetsanopoulos, Color Image Processing and Applications. Springer Verlag, 2000.

[4] N. Kehtarnavaz, H.J Oh, and Y. Yoo, "Color filter array interpolation using color correlations and directional derivatives," Journal of Electronic Imaging, to appear 2003.

[5] J. Adams, "Design of practical color filter array interpolation algorithms for digital cameras," Proc. SPIE, vol. 3028, pp. 117-125, Feb. 1997.

[6] S.C. Pei and I.K. Tam, "Effective color interpolation in CCD color filter arrays using signal correlation," IEEE Trans. Circuits and Systems for Video Technology, vol. 13, no. 6, pp. 503-513, June 2003.

[7] B. Gunturk, Y. Altunbasak, and R. Mersereau, "Color plane interpolation using alternating projections," IEEE Trans. Image Processing, vol. 11, no.9, pp. 997-1013, September 2002.

[8] P. Longere, Z. Xuemei, P.B. Delahunt, and D.H. Brainard, "Perceptual assessment of demosaicing algorithm performance," Proceedings of the IEEE, vol. 90, no. 1, pp. 123-132, Jan. 2002.

[9] W.T. Freeman, "Median filter for reconstructing missing color samples," U.S. Patent 5373 322, 1988.

[10] B.S. Hur and M.G. Kang, "High definition color interpolation scheme for progressive scan CCD image sensor," IEEE Trans. Consumer Electronics, vol. 47, no. 2, pp. 179-186, February 2001.

[11] J.F. Hamilton and J.E. Adams, "Adaptive color plane interpolation in single sensor color electronic camera," U.S. Patent 5629 734, 1997.

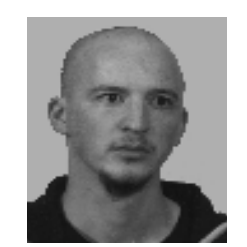

Rastislav Lukac received a Diploma in Telecommunications with honors in 1998 and a Ph.D. in 2001, both at the Technical University of Kosice, Slovak Republic. From February 2001 to August 2002 he was an assistant professor at the Department of Electronics and Multimedia Communications at the Technical University of Kosice. Since August 2002 he is a researcher in Slovak Image Processing Center in Dobsina, Slovak Republic. From January 2003 to March 2003 he was a postdoc at Artificial Intelligence \& Information Analysis Lab at the Aristotle University of Thessaloniki, Greece. In 2003, he was awarded the NATO Science Fellowship. Since May 2003 he has been a post-doctoral fellow at the Edward S. Rogers Sr. Department of Electrical and Computer Engineering at the University of Toronto in Toronto, Canada.

Dr. Lukac is a member of the IEEE Signal Processing Society. He is an active member of Review and Program Committees at various European conferences and a reviewer for various scientific journals. Recently, his research interests include nonlinear digital filters, image sharpening and analysis, color image processing, CFA interpolation/zooming and digital camera image processing, image sequence processing, multimedia, and the use of Boolean functions, permutation theory and artificial intelligence in filter design.

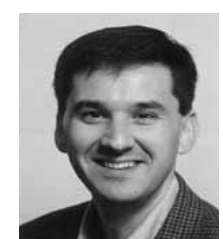

Konstantinos N. Plataniotis received the B. Engineering degree in Computer Engineering from the Department of Computer Engineering and Informatics, University of Patras, Patras, Greece in 1988 and the M.S and Ph.D degrees in Electrical Engineering from the Florida Institute of Technology (Florida Tech), Melbourne, Florida in 1992 and 1994 respectively. He was affiliated with the Computer Technology Institute (C.T.I), Patras, Greece from 1989 to 1991 . He was with the Digital Signal $\backslash \&$ Image Processing Laboratory, Department of Electrical and Computer Engineering University of Toronto, from 1995 to 1997. >From August 1997 to June 1999 he was an Assistant Professor with the School of Computer Science at Ryerson University. While at Ryerson Prof. Plataniotis served as a lecturer in 12 courses to industry and Continuing Education programs. Since 1999 he has been with the University of Toronto. He is currently an Assistant Professor at the Edward S. Rogers Sr. Department of Electrical \& Computer Engineering where he researches and teaches adaptive systems and multimedia signal processing.

Dr. Plataniotis is the Bell Canada Junior Chairholder in Multimedia and a Nortel Institute for Telecommunications Associate. He co-authored, with A.N. Venetsanopoulos, a book on "Color Image Processing \& Applications", Springer Verlag, May 2000, ISBN 3-540-66953-1, he is a contributor to four books, and he has published more than 200 papers in refereed journals and conference proceedings in the areas of multimedia signal processing, image processing, adaptive systems, communications systems and stochastic estimation.

Dr. Plataniotis is a Senior Member of IEEE, a past member of the IEEE Technical Committee on Neural Networks for Signal Processing, and the Technical Co-Chair of the Canadian Conference on Electrical and Computer Engineering, CCECE 2001, May 13-16, 2001, and CCECE 2004, May 2-5 2004.

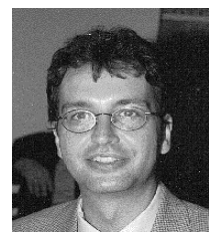

Dimitrios Hatzinakos received the Diploma degree from the University of Thessaloniki, Greece, in 1983, the M.A.Sc degree from the University of Ottawa, Canada, in 1986 and the Ph.D. degree from Northeastern University, Boston, MA, in 1990, all in Electrical Engineering. Currently, he is a Professor and Chair of the Communications Group at the Department of Electrical and Computer Engineering, University of Toronto, Canada.

His research interests are in the area of digital communications and signal processing with applications to wireless communications, image processing and multimedia. He has organized and taught many short courses on modern signal processing frameworks and applications devoted to continuing engineering education and given numerous seminars in the area of blind signal deconvolution. He is author/co-author of more than 100 papers in technical journals and conference proceedings and he has contributed to 5 books in his areas of interest. His experience includes consulting through Electrical Engineering Consociates Ltd. and contracts with United Signals and Systems Inc., Burns and Fry Ltd., Pipetronix Ltd., Defense Research Establishment Ottawa (DREO), Vaytek Inc., and Vivosonic Inc.

$\mathrm{He}$ is an Associate Editor for the IEEE Transactions on Signal Processing since July 1998 and the Guest Editor for the special issue of Signal Processing, Elsevier, on Signal Processing Technologies for Short Burst Wireless Communications scheduled to appear in Fall 2000. He was a member of the IEEE Statistical Signal and Array Processing Technical Committee (SSAP) from 1992 till 1995 and Technical Program co-Chair of the 5th Workshop on Higher-Order Statistics in July 1997. He is a senior member of the IEEE and member of EURASIP, the Professional Engineers of Ontario (PEO), and the Technical Chamber of Greece.

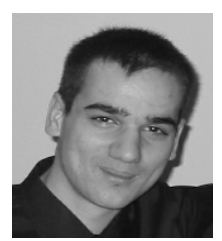

Marko Aleksic was born in Belgrade, Yugoslavia in 1982. Currently he is completing his final year of Engineering Science at the University of Toronto with undergraduate thesis focusing on color filter array interpolation and camera image processing. 